\title{
Disease characteristics and treatment patterns of Chinese patients with metastatic colorectal cancer: a retrospective study using medical records from China
}

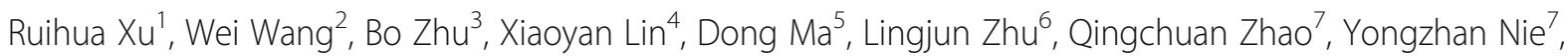
Xiaohong Cai ${ }^{8}$, Qi Li ${ }^{9}$, Weijia Fang ${ }^{10}$, Hongyan $\mathrm{Li}^{11}$, Ning Wang ${ }^{11}$, Yun Chen ${ }^{11}$, Cike Peng ${ }^{11^{*}}$, Honghao Fang ${ }^{12}$ and Lin Shen ${ }^{13}$

\begin{abstract}
Background: Colorectal cancer (CRC) is the third most prevalent cancer in China but few large-scale studies were conducted to understand CRC patients. The current study is aimed to gain a real-world perspectives of CRC patients in China.

Methods: Using electronic medical records of sampled patients between 2011 and 2016 from 12 hospitals in China, a retrospective cohort study was conducted to describe demographics and disease prognosis of CRC patients, and examine treatment sequences among metastatic CRC (mCRC) patients. Descriptive, comparative and survival analyses were conducted.
\end{abstract}

Results: Among mCRC patients (3878/8136, 48\%), the fluorouracil, leucovorin, and oxaliplatin (FOLFOX) and other oxaliplatin-based regimens were the most widely-used first-line treatment (42\%). Fluorouracil, leucovorin, irinotecan (FOLFIRI) and other irinotecan-based regimens dominated the second-line (40\%). There was no a dominated regimen for the third-line. The proportion of patients receiving chemotherapy with targeted biologics increased from less than $20 \%$ for the first- and second- lines to $34 \%$ for the third-line $(p<0.001)$. The most common sequence from first- to second-line was from FOLFOX and other oxaliplatin-based regimens to FOLFIRI and other irinotecan-based regimens (286/1200, 24\%).

Conclusions: Our findings reflected a lack of consensus on the choice of third-line therapy and limited available options in China. It is evident o continue promoting early CRC diagnosis and to increase the accessibility of treatment options for mCRC patients. As the only nationwide large-scale study among CRC and mCRC patients before more biologics became available in China, our results can also be used as the baseline to assess treatment pattern changes before and after more third-line treatment were approved and covered into the National Health Insurance Plan in China between 2017 and 2018.

Keywords: Treatment patterns, Colorectal cancer, Medical records, Real-world evidence, China

\footnotetext{
* Correspondence: Peng_ci_ke@lilly.com

${ }^{11}$ Eli Lilly and Company China Affiliate, No. 288 Shi Men Yi Lu, Jing'an District,

Shanghai 200041, China

Full list of author information is available at the end of the article
}

(c) The Author(s). 2020 Open Access This article is distributed under the terms of the Creative Commons Attribution 4.0 International License (http://creativecommons.org/licenses/by/4.0/), which permits unrestricted use, distribution, and reproduction in any medium, provided you give appropriate credit to the original author(s) and the source, provide a link to the Creative Commons license, and indicate if changes were made. The Creative Commons Public Domain Dedication waiver (http://creativecommons.org/publicdomain/zero/1.0/) applies to the data made available in this article, unless otherwise stated. 


\section{Background}

Colorectal cancer (CRC) is the third most common cancer in China, with 370,000 new cases in year 2014, comprising 9.73\% of all cancers [1]. CRC is also one of the leading causes of cancer deaths in China. Risks of CRC increase with age, especially after age 35 , and reach a peak among people aged 80-84years old [2]. The age-standardized incidence rate by Chinese standard population (ASIRC) was estimated to be 14.20 per 100,000 in 2012 , increasing to 17.45 per 100,000 in 2013 and to 17.76 per 100,000 in $2014[1,2]$. Besides age, there has been evidence that a diet high in fats and low in fruits and vegetables increases the risk of developing CRC $[3,4]$. Because of the rapidly aging population and in the increasing fat intake in China, CRC incidence is expected to continue increasing. Moreover, the National Central Cancer Registry (NCCR) showed that prognosis of CRC was much poorer in China compared with developed countries [5].

Despite the significant disease burden, there is limited information on CRC patient characteristics and disease patterns in China. The NCCR synthesizes data collected from local registries in China and reports basic statistics such as incidence and mortality by key risk groups at the national level [6]. Population-based studies were usually conducted in particular geographic regions [7-10], among a specific group of patients [8], or out-of-date [11]. For CRC patients with metastatic colorectal cancer (mCRC), treatments can be complex. As multiple chemotherapeutic and targeted biologic agents emerged, treatment patterns and sequences for mCRC patients have significantly evolved over the past decade [12]. Several studies have reported the complex and changing treatment pattern among $\mathrm{mCRC}$ patients in the United States [13-16], Canada [17], and some European countries $[18,19]$. However, there is a lack of key real-world evidence on the clinical characteristics and treatment patterns of $\mathrm{CRC} / \mathrm{mCRC}$ patients in China.

Thus, this study was designed to describe baseline characteristics of CRC and $\mathrm{mCRC}$ patients, to investigate prognosis in CRC patients, as well as to understand treatment patterns and sequences in mCRC patients using a multi-center oncology database [20]. Findings from this study can be used as evidence to inform clinical management of CRC and mCRC patients.

\section{Methods}

\section{Data source}

This study analyzed data drawn from a multi-center oncology database, which gathered information from electronic medical records (EMRs) of multiple tertiary hospitals in China. With a large volume of patient-level data of patient sample and a wide geographic coverage, this database provides a platform for conducting a retrospective database study among CRC and mCRC patients.
A total of 12 tertiary hospitals from eight provinces were selected across China. Data between January 1, 2011 and September 30, 2016 were extracted, including information on patient baseline characteristics and detailed diagnosis and treatment-related information during each inpatient visit.

\section{Study population}

Patients with primary diagnosis dates after January 1, 2011 and aged 18 years old and above at primary diagnosis were included. Primary diagnosis dates (baseline) were defined as the first clinical or pathological diagnosis dates recorded in the selected hospital's EMRs, whichever occurred earlier. If patients were previously diagnosed with CRC outside the selected hospitals, the initial diagnosis dates, if available, were used as the primary diagnosis dates. As a previous phase III clinical trial suggested that the maximum period of the 3rd line treatment usage was nine months [21], in this study, patients who started third-line chemotherapy after January 1, 2016 were excluded to allow a minimum nine-month observation period for the entire third-line chemotherapy to be documented.

Patients with mCRC, as a subgroup of CRC patients, included those who were classified as TNM stage IV [22] at primary diagnosis, and those whose tumor metastasized before the database lock (September 30, 2016).

\section{Study variables}

Patient demographic and clinical characteristics at primary diagnosis were analyzed for the CRC and the mCRC subgroup. As the first appearance of metastases was not recorded in EMRs, metastases were defined by the adoption of palliative chemotherapy. Recurrence rates were assessed at 1-year, 2-year and 3-year postindex dates and compared by TNM stage and primary tumor site. Disease-free survival was defined as the time between the primary diagnosis date and the first documentation of recurrent of local or regional tumor, or deaths or the last record date in the EMR database, whichever occurred earlier. Treatment patterns of first-, second- and third-line palliative chemotherapy were also analyzed, with regimens and cycles reported. Chemotherapeutic lines were determined by physicians and recorded in EMRs.

\section{Statistics}

For continuous variables, mean, standard deviation (SD), median, interquartile range (IQR), minimum (min) and maximum $(\max )$ values were presented as appropriate; for categorical variables, number of missing values, frequency distribution and percentage were presented. Missing data were not included in percentage calculations. Chi-square tests were used to compare categorical 
variables and Kruskal-Wallis tests for continuous variables. Recurrence rates were compared by TNM stage and primary tumor site using Fisher's exact tests instead of Chi-square tests due to a smaller sample size. Cumulative probabilities of disease-free survival by TNM stage were estimated using the Kaplan-Meier method, and comparisons were performed with the log-rank test. All statistical analyses were performed using STATA version 14.0 (Stata Corporation, Texas, USA). Two-sided tests with a significance level of 0.05 were applied.

\section{Results}

\section{Patient flow}

Data of 8246 CRC patients identified from the selected hospitals during the study period were extracted and screened. Among them, 39 patients had missing values or were under 18 years old at primary diagnosis, and 71 patients started third-line palliative chemotherapy after January 1, 2016. After excluding these 110 patients, 8136 CRC patients were included (Fig. 1). Over one-third $(2963 / 8136,36 \%)$ of these patients were at TNM stage IV at primary diagnosis, and an additional 915 (11\%) patients metastasized during the observation period. Thus, 3878 (47\% of 8246$)$ mCRC patients were identified.

\section{Baseline characteristics for CRC patients}

The mean age of CRC patients was 59 (SD 13) years old and $60 \%$ of them were males. Most of the patients (87\%) presented in internal medicine departments. The size of primary tumor was only available among 36\% (2926/
8136) patients with a mean of $4.48 \mathrm{~cm}$ (SD 2.05). The KRAS mutation status testing rate was only $25 \%$. The majority of CRC patients had left-sided primary tumor sites (71\%) and were physically well (98\%) at baseline with an Eastern Cooperative Oncology Group Performance Status (ECOG PS) scoring of 0 or 1 (Table 1). Of the 8136 CRC patients, 6764 (83\%) had TNM classification records at primary diagnosis. Among them, 7.2, 19, 30 and $44 \%$ were at stages I, II, III and IV, respectively. Liver was the most common metastatic site (52\%), followed by lung (27\%) (Fig. 2).

\section{Disease recurrent risks for CRC patients with radical surgeries}

For patients who underwent radical surgeries, the cumulative recurrence rate at year 1,2, and 3 was 8.9, 16 and $30 \%$, respectively. By different baseline TNM stage, an upward trend could be seen from baseline stage I to stage III for all 1-, 2- and 3-year recurrence rates $(p<$ 0.001, Additional file 1: Table S1). Recurrence rates did not differ significantly between left- and right-sided primary tumor sites (Additional file 1: Table S2). The log-rank test showed that differences across TNM stagespecific, disease-free survival curves were of statistical significance $(p<0.001$, Fig. 3$)$.

\section{Baseline characteristics for $\mathrm{mCRC}$ patients}

The mean age of mCRC patients at the primary diagnosis date were 57 years old (SD 12). There were more males in mCRC patients (62\%) and most (92\%) presented in internal

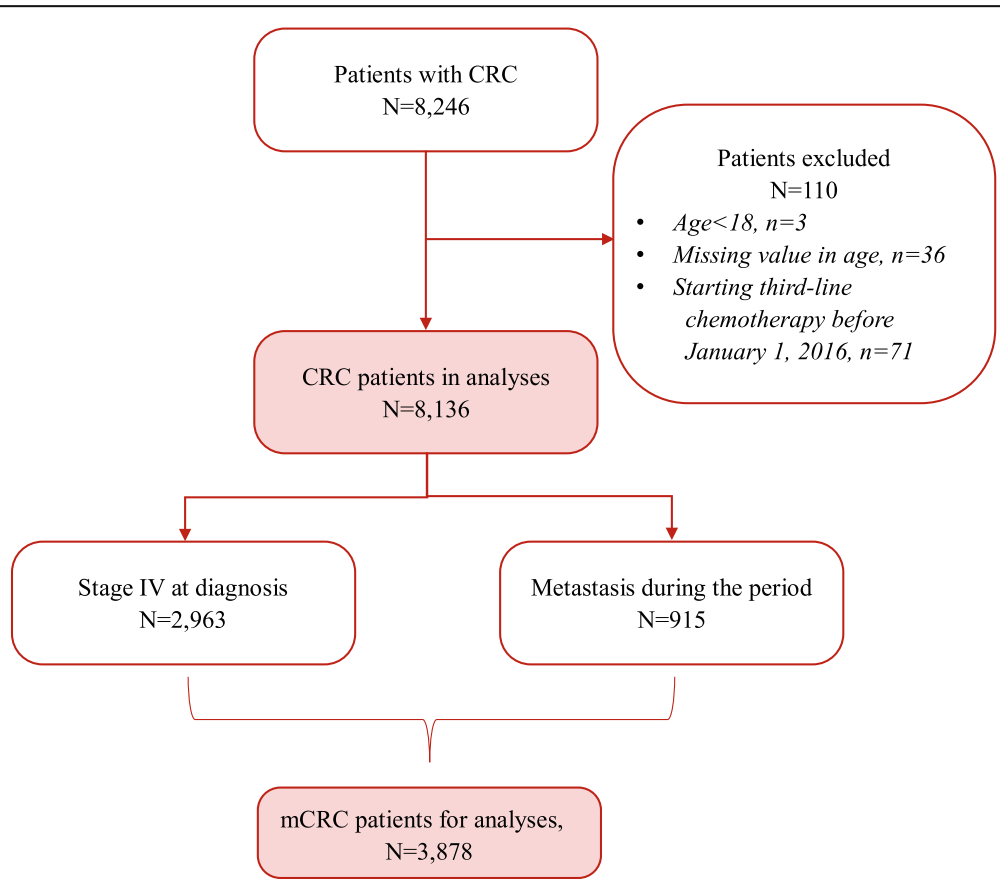

Fig. 1 Patient flow 
Table 1 Baseline characteristics of CRC and mCRC patients

\begin{tabular}{|c|c|c|}
\hline Characteristics & $\begin{array}{l}\text { CRC patients } \\
(N=8136)\end{array}$ & $\begin{array}{l}\text { mCRC patients } \\
(N=3878)\end{array}$ \\
\hline \multicolumn{3}{|l|}{ Age at diagnosis, year } \\
\hline Mean (SD) & $59(13)$ & $57(12)$ \\
\hline Median (IQR) & $60(50-68)$ & $58(49-66)$ \\
\hline Min - max & $18-96$ & $18-96$ \\
\hline \multicolumn{3}{|l|}{ Age group, n (\%) } \\
\hline $18-49$ & $1883(23 \%)$ & $1075(28 \%)$ \\
\hline $50-59$ & $2035(25 \%)$ & $1067(28 \%)$ \\
\hline $60+$ & $4218(52 \%)$ & $1736(45 \%)$ \\
\hline \multicolumn{3}{|l|}{ Gender, n (\%) } \\
\hline Female & $3233(40 \%)$ & $1472(38 \%)$ \\
\hline Male & $4903(60 \%)$ & $2406(62 \%)$ \\
\hline \multicolumn{3}{|c|}{ Department of practice, $n$ (\%) } \\
\hline Internal medicine & $7074(87 \%)$ & $3584(92 \%)$ \\
\hline Surgery & $1062(13 \%)$ & $294(7.6 \%)$ \\
\hline \multicolumn{3}{|c|}{ Hospital geographic region, $n(\%)$} \\
\hline North & $489(6 \%)$ & $416(11 \%)$ \\
\hline South & $4972(61 \%)$ & $2258(58 \%)$ \\
\hline East & $533(6.6 \%)$ & $463(12 \%)$ \\
\hline Midwest & $2142(26 \%)$ & $741(19 \%)$ \\
\hline \multicolumn{3}{|c|}{ Primary tumor size at diagnosis, centimeters } \\
\hline Missing, $n(\%)$ & $2926(36 \%)$ & $2116(55 \%)$ \\
\hline Mean (SD) & $4.48(2.05)$ & $4.52(2.09)$ \\
\hline Median (IQR) & $4(3-5.5)$ & $4(3-5.5)$ \\
\hline \multicolumn{3}{|l|}{ Mutation status, n (\%) } \\
\hline KRAS MUT & $794(10 \%)$ & $525(14 \%)$ \\
\hline KRAS WT & $1246(15 \%)$ & $805(21 \%)$ \\
\hline Unknown & $6096(75 \%)$ & $2548(66 \%)$ \\
\hline \multicolumn{3}{|c|}{ Primary tumor site at diagnosis, $n(\%)$} \\
\hline Left-sided & $5751(71 \%)$ & $2659(69 \%)$ \\
\hline Right-sided & $1767(22 \%)$ & $879(23 \%)$ \\
\hline Colorectal NOS & $618(7.6 \%)$ & $340(8.8 \%)$ \\
\hline \multicolumn{3}{|c|}{ ECOG PS at diagnosis, $n(\%)$} \\
\hline Missing, $\mathrm{n}$ & 2386 & 1470 \\
\hline 0 & $724(12 \%)$ & $429(18 \%)$ \\
\hline 1 & $4912(86 \%)$ & 1919 (80\%) \\
\hline 2 & $65(1.1 \%)$ & $51(2.1 \%)$ \\
\hline 3 & $11(0.19 \%)$ & $7(0.29 \%)$ \\
\hline 4 & $4(0.07 \%)$ & $2(0.08 \%)$ \\
\hline
\end{tabular}

Note: $C R C$ colorectal cancer, $m C R C$ metastatic colorectal cancer, $S D$ standard deviation, IQR: interquartile range, min minimum, max maximum, MUT mutation, WT wild-type, NOS not otherwise specified, ECOG PS Eastern Cooperative Oncology Group Performance Status medicine departments. The mean size of the primary tumor was $4.52 \mathrm{~cm}$ (SD 2.09). The KRAS mutation status testing rate was $35 \%$. The majority of CRC patients had left-sided primary tumor sites (69\%) and were physically well (98\%) at baseline (Table 1).

\section{Treatment patterns for $\mathrm{mCRC}$ patients with palliative chemotherapy}

Among the 3878 mCRC patients, 79\% (3063) had records on first-line treatment of palliative chemotherapy, 1281 had records on second-line treatment, and $404 \mathrm{had}$ records on third-line treatment. Fluorouracil, leucovorin, and oxaliplatin (FOLFOX) and other oxaliplatin-based regimens were the most frequently administered (1275/ $3063,42 \%$ ) in first-line, followed by fluorouracil, leucovorin, and irinotecan (FOLFIRI) and other irinotecanbased regimens (25\%) (Fig. 4a). Usage of FOLFIRI and other irinotecan-based regimens increased remarkably to $40 \%$ in second-line, which dominated treatment of this line (Fig. 4b). Correspondingly, FOLFOX and other oxaliplatin-based regimens decreased to $21 \%$ in secondline. Less than one-sixth of patients received targeted biologics in combination with chemotherapy (16 and 13\% in first- and second-line, respectively). Among these patients, the majority received bevacizumab (312/418, 75\% and $124 /$ $162,77 \%$ in first- and second-line, respectively). The proportion of patients receiving combination therapy with targeted biologics increased dramatically to $34 \%$ in thirdline treatment (137/404), and bevacizumab was still the dominant choice (79/137, 58\%) (Fig. 4c).

Treatment duration decreased in later lines of treatment. Median cycles were 5 in first-line, 3 in second-line and reduced to 2 in third-line treatment. In addition, patients receiving combination therapy with targeted biologics had longer median cycles than chemotherapy alone in all three lines of treatment ( 6 vs. 4,6 vs. 3 and 3 vs. 2 in the first-, second- and third-line, respectively) (Fig. 4d).

\section{Treatment sequences for $\mathrm{mCRC}$ patients with palliative chemotherapy}

In total, 1200 patients had records on both first- and second-line of treatments, of whom 404 patients had records on third-line of treatments.

Four hundred and ninety-five patients who received FOLFOX and other oxaliplatin-based regimens in firstline treatment changed to second-line, accounting for 39\% (495/1275) of all patients starting treatment with this type of regimen. Among them, the majority (286/495, 58\%) changed to FOLFIRI and other irinotecan-based regimens, which was also the most common sequence between first- and second-line treatments (286/1200, 24\%). FOLFOX and other oxaliplatin-based regimens were somehow re-introduced to a small proportion of these patients $(65 / 495,13 \%)$ in second-line treatment. 


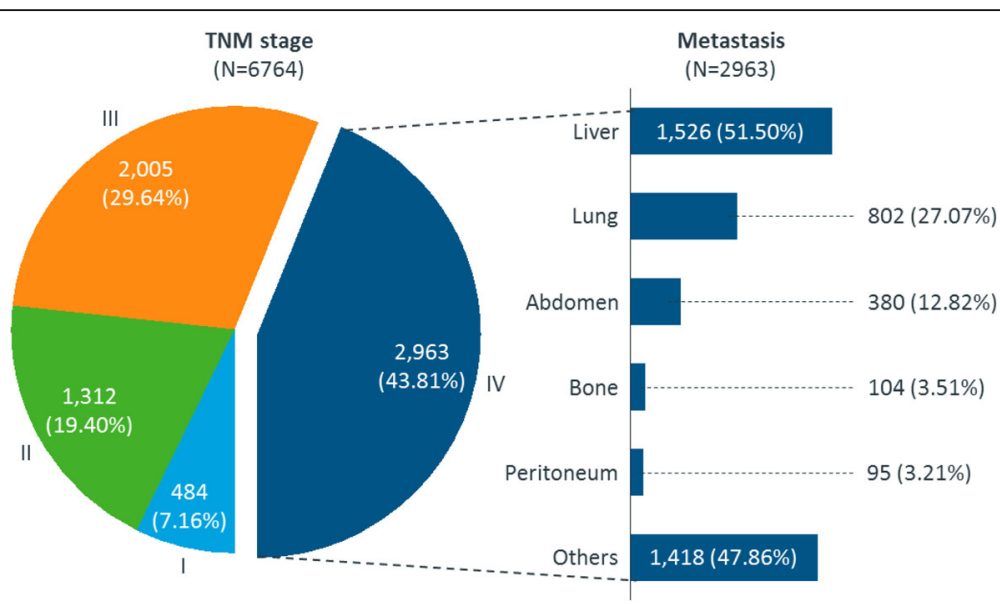

Fig. 2 TNM stage and metastatic status at diagnosis

The second most common sequence between firstand second-line treatments was moving from FOLFIRI and other irinotecan-based regimens to FOLFOX and other oxaliplatin-based regimens (128 out of $1200,11 \%)$.

A total of 994 patients receiving chemotherapy alone in first-line treatment proceeded to second-line, accounting for $39 \%$ of all those received chemotherapy alone. One in ten of them $(101 / 994,10 \%)$ received targeted biologics in combination with chemotherapy as their second-line treatment.

Among the 275 patients receiving the dominant treatment choice in second-line, i.e., FOLFIRI and other irinotecan-based regimens, 151 (55\%) proceeded to thirdline. The most common sequence from the second-line was to FOLFOX and other oxaliplatin-based regimens (36/ 151, 24\%), followed by to other chemotherapy alone (34/ 151, 23\%). A total of 354 patients receiving chemotherapy alone in second-line proceeded to third-line, accounting for $32 \%$ of all those received chemotherapy alone. Among

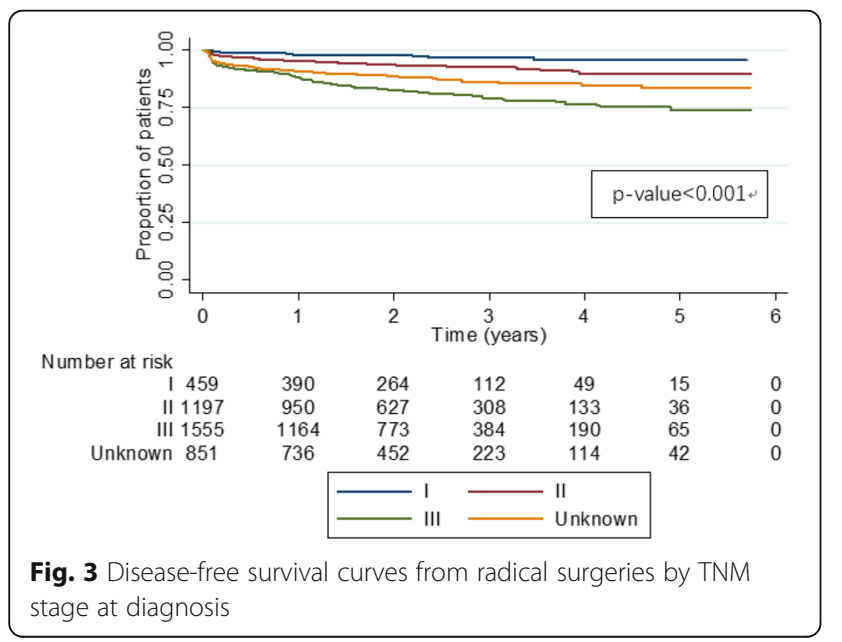

these 354 patients, over a quarter $(105 / 354,30 \%)$ added targeted biologics on top of chemotherapy, which also constituted a large proportion of total patients receiving combination therapy in this line of treatment (105/137, 77\%). Figure 5 demonstrates treatment sequences of palliative chemotherapy from first- to third-line treatments.

\section{Discussion}

To our knowledge, this is the first nationwide large-scale study on CRC and mCRC patient characteristics and treatment sequences in China. We described patient demographics and clinical characteristics, which provided a comprehensive and updated picture of Chinese CRC and mCRC patients in China. Analysis results on prognosis after radical surgeries and clinical practice in palliative chemotherapy can be used as real-world evidence to inform the management of CRC and mCRC. Importantly, our results demonstrated no dominant choice in the third-line therapy. This reflects a lack of consensus on the choice of third-line therapy in China and an urgent need to develop national guidelines on clinical practice.

In this study, the mean age of CRC and mCRC patients diagnosed during 2011 to 2016 was 59 and 57 years old, respectively. For both CRC and mCRC patients, there were more male CRC patients than females. These findings are not surprising compared to previous published reviews and regional studies in China [7-9]. It has been reported that estrogen could prevent CRC [23], which may explain the relatively small proportion of female patients.

In this study, approximately $30 \%$ of patients presented with TNM stage III and about $40 \%$ presented with TNM stage IV at diagnosis. The percentage of patients diagnosed with TNM stage III is generally in line with previous findings which reported a range of $30-40 \%$; while, the percentage of patients identified with stage IV is larger than that reported in previous studies which is 
A First-line treatment $(\mathrm{n}=3,063)$

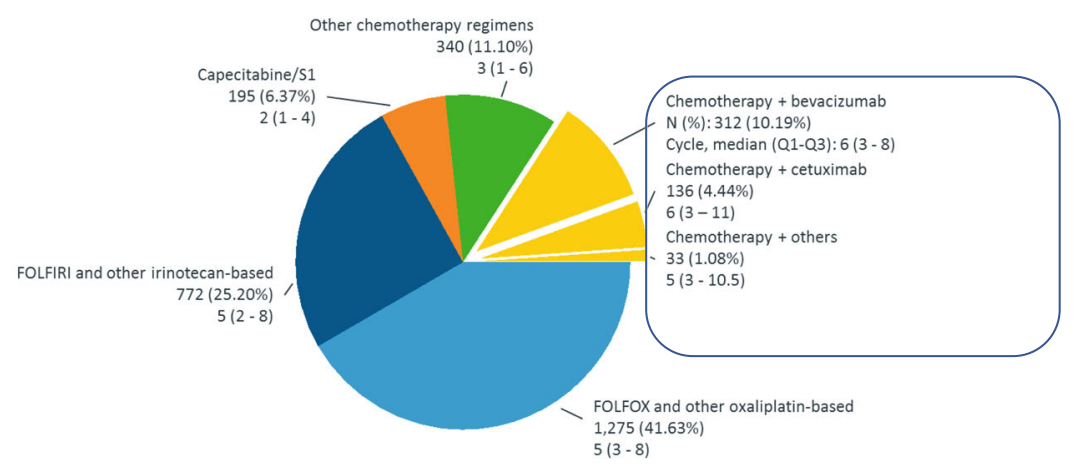

B Second-line treatment $(\mathrm{n}=1,281)$
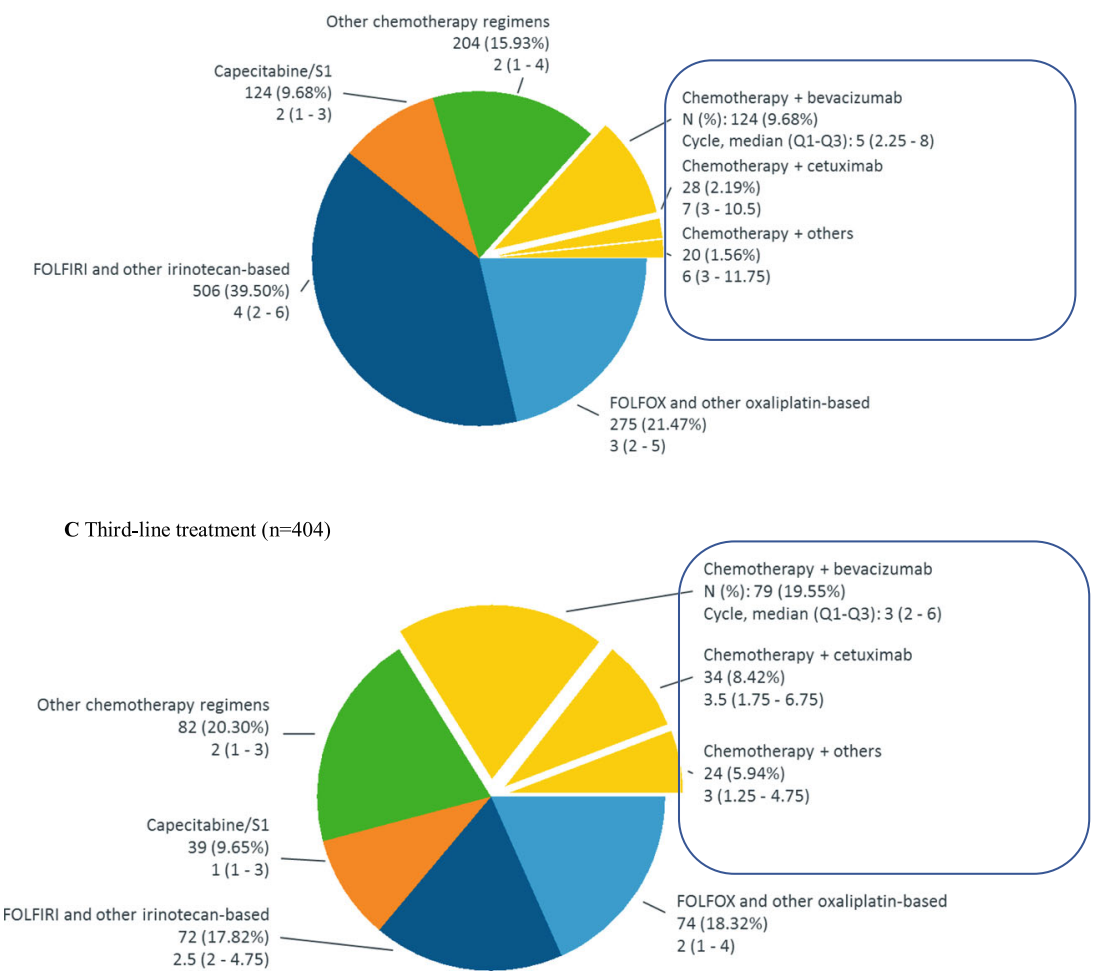

D Number of patients and treatment cycles by chemotherapy alone and combination therapy in each line of treatment

\begin{tabular}{lll}
\hline & \multicolumn{1}{c}{$\mathbf{N}(\mathbf{( \% )}$} & \multicolumn{1}{c}{$\begin{array}{c}\text { Cycle, } \\
\text { median (IQR) }\end{array}$} \\
\hline First-line & $\mathbf{3 , 0 6 3 ( 1 0 0 \% )}$ & $\mathbf{5 ( 2 - 8 )}$ \\
Chemotherapy alone & $2,582(84 \%)$ & $4(2-7)$ \\
Chemotherapy + targeted biologics & $481(16 \%)$ & $6(3-9)$ \\
Second-line & $\mathbf{1 , 2 8 1 ( 1 0 0 \% )}$ & $\mathbf{3 ( 1 - 6 )}$ \\
Chemotherapy alone & $1,109(87 \%)$ & $3(1-5)$ \\
Chemotherapy + targeted biologics & $172(13 \%)$ & $6(3-9)$ \\
Third-line & $\mathbf{4 0 4 ( 1 0 0 \% )}$ & $\mathbf{2 ( 1 - 4 )}$ \\
Chemotherapy alone & $267(66 \%)$ & $2(1-3)$ \\
Chemotherapy + targeted biologics & $137(34 \%)$ & $3(2-6)$ \\
\hline IQR: interquartile range & &
\end{tabular}

Fig. 4 Treatment patterns of palliative chemotherapy for $\mathrm{mCRC}$ patients 


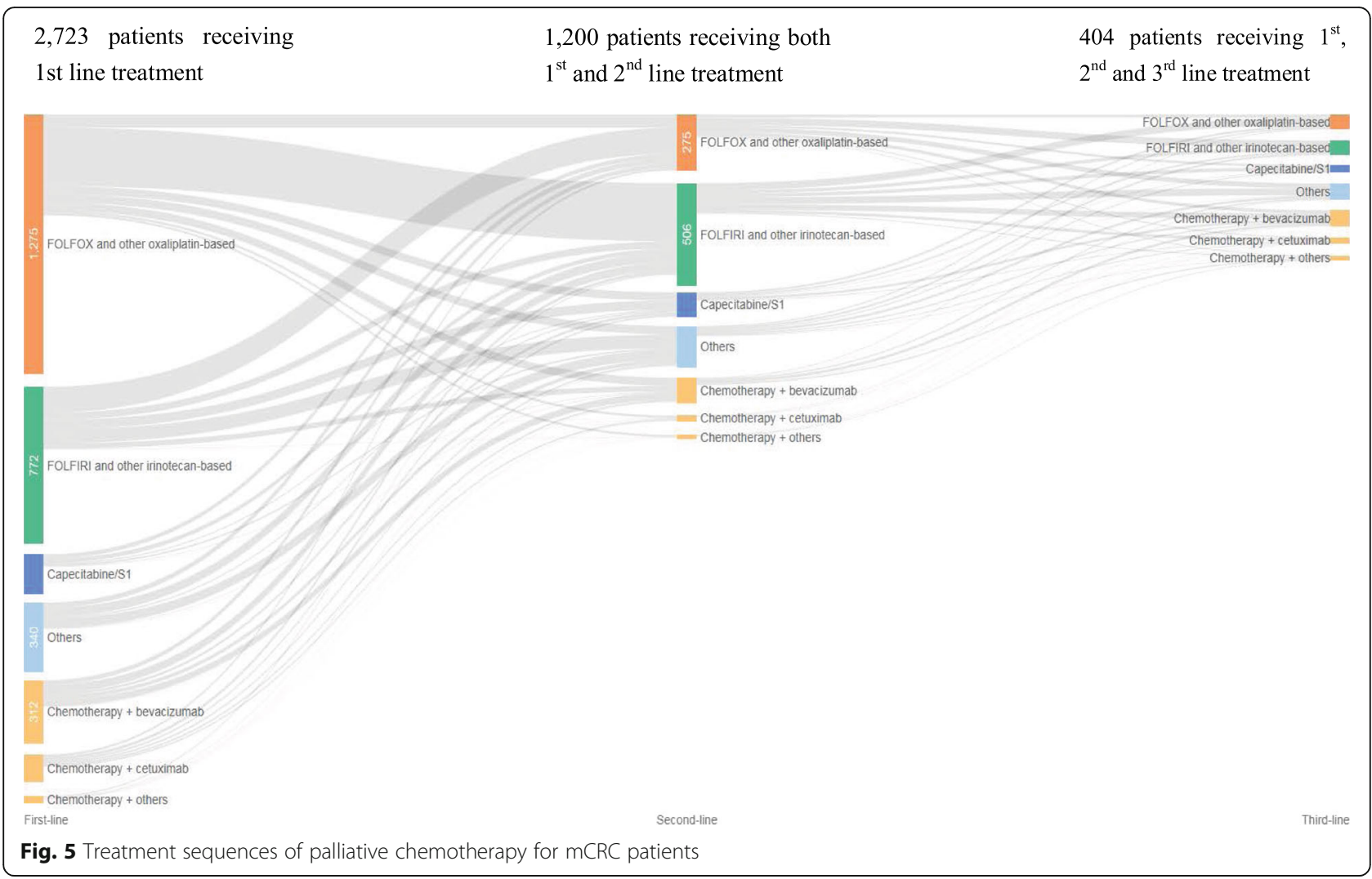

ranged $20-33 \%$ [24, 25]. The inconsistence on the percentage of patients diagnosed with stage IV across studies may be due to the differences in study samples, e.g. the current study included higher proportion of patients aged 60 years and over than other studies. It is also possible that the early detection of colorectal cancer in China is not as prevalent as in other countries. A decrease in the proportion of patients with advanced stage CRC may be expected if an early detection program can be implemented [24].

Most patients in this study did not have their KRAS mutation status tested. Although KRAS mutation analysis may provide additional useful information on risk stratification in colorectal cancer, the predictive value of KRAS mutation for non-response to chemotherapy is still questioning [23]. Previous studies found that the expression of KRAS is associated with recurrence, survival and benefit of adjuvant chemotherapy [26]. Future studies on the predictive value of KRAs in Chinese CRC patients are required.

As expected, the proportion of right-sided CRC (22\%) found in this study was higher than that reported in the 1990s (15\%) and 1980s (11\%) [11]. A similar rightward shift in the primary tumor site of CRC has also been reported in North America, the United Kingdom, Japan and Northern Ireland [27-31], and it is associated with aging [32]. Our study has also found that among patients after radical surgeries, the primary tumor site was not associated with disease-free survival. This is supported by the finding from a most recent study which included 4426 Chinese patients with stage I, II and III CRC [33]. Patient at earlier stages had a significant improvement in survival. This finding underlines the importance of early diagnosis and increased awareness of CRC in China [34, 35].

Our study found that FOLFOX and other oxaliplatinbased regimens dominated first-line while FOLFIRI and other irinotecan-based regimens dominated second-line. This is consistent with findings from other studies. For example, a study with 1655 adult mCRC patients in the US reported that about $40 \%$ of patients received FOLFOX in first-line therapy, and about $26 \%$ of patients received FOLFIRI in second-line therapy [15]. Another US based study reported similar findings [36]. As to third-line therapy, there was no dominated treatments were identified in China. Importantly, targeted biologics were not frequently used in China until third-line treatment, treatment cycles in third-line was short and some patients moved back to their previously used therapy. In contrast, in the US, the most common third line treatment regimens are EGFR-containing therapies, such as combination of cetuximab and irinotecan, panitumumab or cetuximab monotherapy [15]. In other countries, such as Canada, although oxaliplatin and irinotecan were also 
the most common chemotherapy backbones for firstand second-line, chemotherapy was usually not used alone $[17,18]$. This may imply poor treatment outcomes of targeted therapy in late lines, and a lack of consensus on both the timing and options for third-line treatment during the study period (2011-2016). Moreover, the current study found that a small proportion of patients treated with FOLFOX in the first-line setting either shifted to capecitabine/S1 or continued to use FOLFOX in the second-line setting, which was controversial to the guidelines for standard of care [37]. This gap between clinical practice and guidelines may be due to patients' preference, economic status, physicians' decisions [37-39]. Implementing education program and providing training courses to physicians as well as raising the public awareness of CRC might help with the treatment decisionmaking.

There have been some major changes in $\mathrm{mCRC}$ treatment, especially in the third line since 2017. For example, Bevacizumab (Avastin ${ }^{\circ}$ ) has been included into the national reimbursement list and Regorafenib (Stivarga ${ }^{\circ}$ ) has been approved by CFDA. On September 5th, 2018, Fruquintinib (Elunate ${ }^{\circ}$ ) was approved in China's market. These new treatment options on market have increased regimen choice and likely expenditures. Despite of that, the optimal use of these agents along with chemotherapy needs further investigation as a phase II multicenter trial reported little objective responses to the combination of bevacizumab and chemotherapies among advanced CRC patients [40]. Further studies on the cost and effectiveness of treatment sequences using real-world data are warranted to provide important and updated evidence for improving patients' survival.

This study had several limitations. Although we had a wide geographic coverage, all selected hospitals were tertiary hospitals located in large cities. In the three-tier healthcare system of China, patients with severe diseases, such as cancer, would mostly be referred to tertiary hospitals in large cities, but nevertheless those who cannot afford such treatments may present elsewhere. Therefore, sampling bias may still present a barrier to understanding the complete picture of CRC and mCRC patients in China. In addition, if patients visited hospitals outside the selected ones during the observation period, those records would not have been captured and therefore could not be analyzed in this study. Moreover, we utilized a multicenter database specialized in oncology, however the study population was a sample of the whole CRC patient population seen for care in the 12 hospitals. There is no evidence that the sampling process and data collection may introduce significant selection bias. Some key time points, such as the appearance of metastasis, were not recorded in EMRs and were estimated using best proxies. Finally, some key information might not be available or well documented in EMR databases in China. For examples, death information was poorly documented and disease progression information was not directly captured. It is not possible to link an EMR database to the National Death Registry to obtain Deaths. Information on confounders, for examples, diet, smoking, alcohol, was not available in the database. Thus, in the current study, we were not able to provide a more comprehensive view of effectiveness of regimens on survival. It is evident that further real-world evidence, especially from Registries or prospective studies, is required.

\section{Conclusions}

In this multi-center, retrospective study of patients with CRC and mCRC, the current status of CRC and mCRC clinical management were investigated. Among mCRC patients, FOLFOX and FOLFIRI were the dominated first-line and second-line therapies, respectively. There was an increasing trend of using targeted biologics in third-line therapy. With new medications approved or included in national reimbursement scheme in China in recent years, the current findings will be useful in exploration of changing trends of therapies for CRC patients in China.

\section{Supplementary information}

Supplementary information accompanies this paper at https://doi.org/10. 1186/s12885-020-6557-5.

Additional file 1: Table S1. Recurrent rates from radical surgeries by TNM stage at diagnosis. Table S2. Recurrent rates from radical surgeries by primary tumor site

\section{Abbreviations}

ASIRC: Age-standardized incidence rate by Chinese standard population; CRC: Colorectal cancer; ECOG PS: Eastern Cooperative Oncology Group Performance Status; EMRs: Electronic medical records; FOLFIRI: Fluorouracil, leucovorin and irinotecan; FOLFOX: Fluorouracil, leucovorin and oxaliplatin; IQR: Interquartile range; Max: Maximum; mCRC: Metastatic colorectal cancer; Min: Minimum; MUT: Mutation; NCCR: National central cancer registry; NOS: Not otherwise specified; SD: Standard deviation; TNM: Tumor, models and metastases; WT: Wild-type

\section{Acknowledgements}

We thank Medbanks for data provision for the current study.

\section{Authors' contributions}

$\mathrm{RX}$ conceived of the presented idea, participated in data collection, article writing and editing. WW, BZ, XL, DM, LZ, QZ, YN, XC, QL and WF carried out data collection, writing-review and editing of the article; HL, NW, YC, CP, HF and LS conducted analysis and reviewed the article. All authors discussed the results and approved the final manuscript.

\section{Author's information}

Not applicable

\section{Funding}

This study was funded by Lilly Suzhou Pharmaceutical Company Limited. Four authors (Hongyan Li, Ning Wang, Yun Chen and Cike Peng) from Lilly Company participated in data analysis and in writing the manuscript. 


\section{Availability of data and materials}

The data underlying the study is from EMR databases in multiple tertiary hospitals in China. It is not publicly available, and restrictions apply to the availability of the data.

\section{Ethics approval and consent to participate}

De-identification of personal information was performed at the data entry stage in the multi-center oncology database. Ethics approvals were obtained for this study from Beijing Cancer Hospital Ethics Committee, Sun Yat-sen University Cancer Center Ethics Committee and Xijing Hospital Ethics Committee. For hospitals that did not require ethics approval as a mandatory, a form of 'Confidential and non-use Agreement' was assigned. The informed consent was waived by the IRBs from all hospitals.

The full names of hospitals that did not require ethics approval as a mandatory were:

The First People's Hospital of Foshan.

Xinqiao Hospital, Third Military Medical University.

Fujian Medical University Union Hospital.

Guangdong General Hospital.

Jiangsu Province Hospital.

Sichuan Cancer Hospital and Institute.

Shanghai General Hospital.

The First Hospital of Zhejiang Province.

\section{Consent for publication}

Not applicable

\section{Competing interests}

The authors declare that they have no competing interests.

\section{Author details}

${ }^{1}$ Sun Yat-sen University Cancer Center, No. 651 Dongfeng East Road, Yuexiu District, Guangzhou 510060, China. ${ }^{2}$ The First People's Hospital of Foshan, No. 81 North Lingnan Road, Chancheng District, Foshan 528000, China. ${ }^{3}$ Xinqiao Hospital, Third Military Medical University, No. 83 Xinqiaozheng Street, Shapingbei District, Chongqing 400037, China. ${ }^{4}$ Fujian Medical University Union Hospital, No. 29 Xinquan Road, Gulou District, Fuzhou 350001, China. ${ }^{5}$ Guangdong General Hospital, No.106 Zhongshan Road, Yuexiu District, Guangzhou 510245, China. ${ }^{6} J i a n g s u$ Province Hospital, No.300 Guangzhou Road, Gulou District, Nanjing 210029, China. ${ }^{7}$ Xijing Hospital, Fourth Military Medical University, No.15 West Road, Xincheng District, 710032, Xi'an, Changle, China. ${ }^{8}$ Sichuan Cancer Hospital and Institute, No.55 Sectional 4, South Renmin Road, Wuhou District, Chengdu 610015, China. ${ }^{9}$ Shanghai General Hospital, No.100 Haining Road, Hongkou District, Shanghai 200080, China. ${ }^{10}$ The First Hospital of Zhejiang Province, No.79 Qingchun Road, Shangcheng District, Hangzhou 310002, China. "11Eli Lilly and Company China Affiliate, No. 288 Shi Men Yi Lu, Jing'an District, Shanghai 200041, China. ${ }^{12}$ IQVIA, No. 968 West Beijing Road, Jing'an District, Shanghai 200063, China. ${ }^{13}$ Beijing Cancer Hospital, No. 52 Fucheng Road, Haidian District, Beijing 100142, China.

Received: 3 July 2019 Accepted: 20 January 2020

\section{Published online: 18 February 2020}

\section{References}

1. Chen W, Zheng R, Zhang S, Zhao P, Zeng H, Zou X. Report of cancer incidence and mortality in China, 2010. Ann Transl Med. 2014 Jul;2(7):61.

2. Du LB, Li HZ, Wang YQ, Zhu C, Zheng RS, Zhang SW, Chen WQ, He J. Report of colorectal cancer incidence and mortality in China, 2013. Zhonghua Zhong Liu Za Zhi. 2017 Sep;39(9):701-6.

3. Haggar FA, Boushey RP. Colorectal cancer epidemiology: incidence, mortality, survival, and risk factors. Clin Colon Rectal Surg. 2009 Nov; 22(04):191-7.

4. Correa Lima MP, Gomes-da-Silva MH. Colorectal cancer: lifestyle and dietary factors. Nutr Hosp. 2005;20(4):235-41.

5. Zeng H, Zheng R, Guo Y, Zhang S, Zou X, Wang N, Zhang L, Tang J, Chen J, Wei K, Huang S. Cancer survival in C hina, 2003-2005: a population-based study. Int J Cancer. 2015 Apr 15;136(8):1921-30.

6. Zheng $\mathrm{R}$, Zeng $\mathrm{H}$, Zhang $\mathrm{S}$, Chen W. Estimates of cancer incidence and mortality in China, 2013. Chin J Cancer. 2017 Dec;36(1):66.
7. Yusup A, Wang HJ, Rahmutula A, Sayim P, Zhao ZL, Zhang GQ. Clinical features and prognosis in colorectal cancer patients with different ethnicities in Northwest China. World J Gastroenterol: WJG. 2013 Nov 7; 19(41):7183.

8. Zhang S, Cui Y, Weng Z, Gong X, Chen M, Zhong B. Changes on the disease pattern of primary colorectal cancers in southern China: a retrospective study of 20 years. Int J Color Dis. 2009 Aug 1;24(8):943-9.

9. Fu JF, Huang YQ, Yang J, Yi CH, Chen $\mathrm{HL}$, Zheng S. Clinical characteristics and prognosis of young patients with colorectal cancer in eastern China. World J Gastroenterol. 2013;19(44):8078.

10. Xu AG, Yu ZJ, Jiang B, Wang XY, Zhong XH, Liu JH, Lou QY, Gan AH. Colorectal cancer in Guangdong Province of China: a demographic and anatomic survey. World J Gastroenterol. 2010;16(8):960.

11. Li M, Gu J. Changing patterns of colorectal cancer in China over a period of 20 years. World J Gastroenterol. 2005;11(30):4685.

12. Martini G, Troiani T, Cardone C, Vitiello P, Sforza V, Ciardiello D, Napolitano S, Della Corte CM, Morgillo F, Raucci A, Cuomo A. Present and future of metastatic colorectal cancer treatment: a review of new candidate targets. World J Gastroenterol. 2017;23(26):4675.

13. Abrams TA, Meyer G, Schrag D, Meyerhardt JA, Moloney J, Fuchs CS. Chemotherapy usage patterns in a US-wide cohort of patients with metastatic colorectal cancer. J Natl Cancer Inst. 2014 Feb 1;106(2):djt371.

14. Parikh RC, Du XL, Morgan RO, Lairson DR. Patterns of treatment sequences in chemotherapy and targeted biologics for metastatic colorectal cancer: findings from a large community-based cohort of elderly patients. DrugsReal World Outcomes. 2016 Mar 1;3(1):69-82.

15. Hess GP, Wang PF, Quach D, Barber B, Zhao Z. Systemic therapy for metastatic colorectal cancer: patterns of chemotherapy and biologic therapy use in US medical oncology practice. J Oncol Pract. 2010 Nov; 6(6):301-7.

16. Zafar SY, Marcello JE, Wheeler JL, Rowe KL, Morse MA, Herndon JE, Abernethy AP. Longitudinal patterns of chemotherapy use in metastatic colorectal cancer. J Oncol Pract. 2009 Aug 20;5(5):228-33.

17. McLean J, Rho YS, Kuruba G, Mamo A, Gilabert M, Kavan T, Panasci L, Melnychuk D, Batist G, Kavan P. Clinical practice patterns in chemotherapeutic treatment regimens for metastatic colorectal cancer. Clin Colorectal Cancer. 2016 Jun 1;15(2):135-40.

18. Zhao Z, Pelletier E, Barber B, Bhosle M, Wang S, Gao S, Klingman D. Patterns of treatment with chemotherapy and monoclonal antibodies for metastatic colorectal cancer in Western Europe. Curr Med Res Opin. 2012 Feb 1;28(2):221-9.

19. Heiman F, Ripellino C, Visentin E. Real world data in oncology: third-and fourth-line treatments administered in metastatic Colon-rectal Cancer (MCRC). Value Health. 2014;17(7):A644.

20. Medbanks. Available from: https://www.medbanks.cn. Accessed 17 June 2019.

21. Li J, Qin S, Xu RH, Shen L, Xu J, Bai Y, Yang L, Deng Y, Chen ZD, Zhong H, Pan $\mathrm{H}$. Effect of fruquintinib vs placebo on overall survival in patients with previously treated metastatic colorectal cancer: the FRESCO randomized clinical trial. JAMA. 2018:319(24):2486-96.

22. Ueno H, Mochizuki H, Akagi Y, Kusumi T, Yamada K, Ikegami M, Kawachi H, Kameoka S, Ohkura Y, Masaki T, Kushima R. Optimal colorectal cancer staging criteria in TNM classification. J Clin Oncol. 2012;30(13):1519-26.

23. Jänne PA, Mayer RJ. Chemoprevention of colorectal cancer. N Engl J Med. 2000;342(26):1960-8.

24. Carrato A. Adjuvant treatment of colorectal cancer. Gastrointest Cancer Res. 2008;2(4 Suppl 2):S42.

25. Kumar S, Burney IA, Zahid KF, Souza PC, Belushi MA, Meki TDMWAFM, Mansour S. Colorectal cancer patient characteristics, treatment and survival in Oman-a single center study. Asian Pac J Cancer Prev. 2015;16(12):4853-8.

26. Bozkurt O, Inanc M, Turkmen E, Karaca H, Berk V, Duran AO, Ozaslan E, Ucar M, Hacibekiroglu I, Eker B, Baspinar O. Clinicopathological characteristics and prognosis of patients according to recurrence time after curative resection for colorectal cancer. Asian Pac J Cancer Prev. 2014;15(21):9277-81.

27. Hutchins G, Southward K, Handley K, Magill L, Beaumont C, Stahlschmidt J, Richman S, Chambers P, Seymour M, Kerr D, Gray R. Value of mismatch repair, KRAS, and BRAF mutations in predicting recurrence and benefits from chemotherapy in colorectal cancer. J Clin Oncol. 2011;29(10):1261-70.

28. Beart RW, Melton $\amalg$, Maruta M, Dockerty MB, Frydenberg HB, O'Fallon WM. Trends in right and left-sided colon cancer. Dis Colon Rectum. 1983;26(6):393-8.

29. Kee F, Wilson RH, Gilliland R, Sloan JM, Rowlands BJ, Moorehead RJ. Changing site distribution of colorectal cancer. BMJ. 1992;305(6846):158. 
30. Jass JR. Subsite distribution and incidence of colorectal cancer in New Zealand, 1974-1983. Dis Colon Rectum. 1991;34(1):56-9.

31. Takada H, Ohsawa T, Iwamoto S, Yoshida R, Nakano M, Imada S, Yoshioka K, Okuno M, Masuya Y, Hasegawa K, Kamano N. Changing site distribution of colorectal cancer in Japan. Dis Colon Rectum. 2002;45(9):1249-54.

32. Kee F, Collins BJ, Patterson CC. Incidence and site distribution of colorectal cancer in Northern Ireland. Ulster Med J. 1990;59(2):155.

33. Liu F, Li C, Jia H, Yang L, Wu Y, Zhao J, Cai S, Zhu J, Xu Y. Is there a prognostic value of tumor location among Chinese patients with colorectal cancer? Oncotarget. 2017;8(24):38682.

34. lida Y, Kawai K, Tsuno NH, Ishihara S, Yamaguchi H, Sunami E, Kitayama J, Watanabe T. Proximal shift of colorectal cancer along with aging. Clin Colorectal Cancer. 2014;13(4):213-8.

35. Leung D, Chow K, Lo S, So W, Chan C. Contributing factors to colorectal cancer screening among Chinese people: a review of quantitative studies. Int J Environ Res Public Health. 2016;13(5):506.

36. Chastek B, Kulakodlu M, Valluri S, Seal BS. Treatment patterns among metastatic colorectal cancer patients by line of therapy and original stage of diagnosis.2012.

37. Zhang Y, Chen Z, Li J. The current status of treatment for colorectal cancer in China: A systematic review. Medicine. 2017 Oct;96(40):e8242.

38. Weeks JC, Cook EF, O'Day SJ, Peterson LM, Wenger N, Reding D, Harrell FE, Kussin P, Dawson NV, Connors AF Jr, Lynn J. Relationship between cancer patients' predictions of prognosis and their treatment preferences. JAMA. 1998;279(21):1709-14.

39. Kutner JS, Vu KO, Prindiville SA, Byers TE. Patient age and cancer treatment decisions: patient and physician views. Cancer Pract. 2000;8(3):114-9.

40. Chen HX, Mooney M, Boron M, Vena D, Mosby K, Grochow L, Jaffe C, Rubinstein L, Zwiebel J, Kaplan RS. Phase II multicenter trial of bevacizumab plus fluorouracil and leucovorin in patients with advanced refractory colorectal cancer: an NCl treatment referral center trial TRC-0301. J Clin Oncol. 2006;24(21):3354-60.

\section{Publisher's Note}

Springer Nature remains neutral with regard to jurisdictional claims in published maps and institutional affiliations.

Ready to submit your research? Choose BMC and benefit from:

- fast, convenient online submission

- thorough peer review by experienced researchers in your field

- rapid publication on acceptance

- support for research data, including large and complex data types

- gold Open Access which fosters wider collaboration and increased citations

- maximum visibility for your research: over $100 \mathrm{M}$ website views per year

At $\mathrm{BMC}$, research is always in progress.

Learn more biomedcentral.com/submissions 